\title{
Apolipoprotein A-I Q[-2]X Causing Isolated Apolipoprotein A-I Deficiency in a Family with Analphalipoproteinemia
}

\author{
Dominic S. Ng, Lawrence A. Leiter, Camilla Vezina, Philip W. Connelly, and Robert A. Hegele
}

Department of Medicine, St. Michael's Hospital, University of Toronto, Ontario M5B 1A6, Canada

\begin{abstract}
We report a Canadian kindred with a novel mutation in the apolipoprotein (apo)A-I gene causing analphalipoproteinemia. The 34-yr-old proband, product of a consanguineous marriage, had bilateral retinopathy, bilateral cataracts, spinocerebellar ataxia, and tendon xanthomata. High density lipoprotein cholesterol (HDL-C) was $<0.1 \mathrm{mM}$ and apoA-I was undetectable. Genomic DNA sequencing of the proband's apoA-I gene identified a nonsense mutation at codon $[-2]$, which we designate as $Q[-2] X$. This mutation causes a loss of endonuclease digestion sites for both BbvI and Fnu4HI. Genotyping identified four additional homozygotes, four heterozygotes, and two unaffected subjects among the first-degree relatives. Q [-2]X homozygosity causes a selective failure to produce any portion of mature apoA-I, resulting in very low plasma level of HDL. Heterozygosity results in approximately half-normal apoA-I and HDL. Gradient gel electrophoresis and differential electroimmunodiffusion assay revealed that the HDL particles of the homozygotes had peak Stokes diameter of $7.9 \mathrm{~nm}$ and contained apoA-II without apoA-I (Lp-AII). Heterozygotes had an additional fraction of $\mathrm{HDL}_{3}$-like particles. Two of the proband's affected sisters had documented premature coronary heart disease. This kindred, the third reported apoA-I gene mutation causing isolated complete apoA-I deficiency, appears to be at significantly increased risk for atherosclerosis. ( $J$. Clin. Invest. 1994. 93:223-229.) Key words: atherosclerosis • genetics $\bullet$ lipoproteins $\bullet$ coronary heart disease $\bullet$ reverse cholesterol transport
\end{abstract}

\section{Introduction}

Plasma HDL level is inversely correlated with the risk of coronary heart disease (CHD) ${ }^{1}$ and has consistently emerged as a powerful predictor of risk (1-5). ApoA-I is the major protein component of HDL. While the actual mechanism for the protective effect of HDL remains poorly understood, a hypothesis that has received much attention relates to the role of HDL in

Address correspondence to Dr. Robert A. Hegele, DNA Research Laboratory, Room 126WA, St. Michael's Hospital West Annex, 38 Shuter Street, Toronto, ON M5B 1A6, Canada.

Received for publication 10 June 1993 and in revised form $19 \mathrm{Au}$ gust 1993.

1. Abbreviations used in this paper: AAL, analphalipoproteinemia; CHD, coronary heart disease; LCAT, lecithin:cholesterol acyltransferase; Lp-AI, HDL with apoA-I only; Lp-AI/ AII, HDL with both apoA-I and apoA-II;

J. Clin. Invest.

(C) The American Society for Clinical Investigation, Inc.

0021-9738/94/01/0223/07 \$2.00

Volume 93, January 1994, 223-229 "reverse cholesterol transport" (6), a major mechanism for removal of excess cholesterol from peripheral tissues. HDL is a heterogeneous class of lipoprotein particles with regard to both size and hydration density and these different classes may have different associations with disease. For example, $\mathrm{HDL}_{2}$, a major density subfraction, may be the crucial component for prevention of CHD (7). However, this has been challenged by a recent prospective study (8). Alternatively, immunoaffinity chromatography can be used to divide HDL particles into two major subfractions: $(a)$ particles with only apoA-I (Lp-AI) and (b) particles with both apoA-I and apoA-II (Lp-AI/AII) (9). Although Lp-AI has been shown to be the component responsible for efficient cholesterol efflux from peripheral cells (10), other data suggest comparable efficiency for both Lp-AI and Lp-AI/AII with regard to efflux (11). Other putative "cardioprotective" roles of HDL are suggested by in vitro evidence of an antioxidant effect on LDL oxidation $(12,13)$ and prevention of the self-aggregation of LDL (14). These latter effects were felt to be directly related to the apoA-I component.

In normal human plasma, $>90 \%$ of apoA-I is found in HDL. The plasma concentration of apoA-I is also a predictive factor of CHD (7). Mature apoA-I is a 243-amino acid single polypeptide chain synthesized by the liver and intestine. The apoA-I gene is localized to the long arm of chromosome 11 and is at the $5^{\prime}$ end of the apoAI-CIII-AIV gene complex. The apoA-I mRNA translates the 267-amino acid prepropeptide, which undergoes intracellular cleavage leading to a 249-amino acid propeptide. The propeptide is secreted and undergoes extracellular proteolysis giving rise to mature apoA-I.

Genetically determined diseases characterized by complete HDL deficiency can help to understand the mechanism of the cardioprotective role of HDL. A number of kindreds with hereditary analphalipoproteinemia (AAL) have known defects in the apoA-I gene (15-23) that result in virtual absence of plasma HDL. In some other kindreds, the molecular defect either does not involve the apoA-I gene $(24,25)$ or has yet to be identified (26-29). Interestingly, despite a nearly complete absence of HDL in some of the kindreds, there is highly variable susceptibility to premature CHD. Possible reasons include the small sample size of each kindred, confounding associated apolipoprotein deficiencies (15-18), and presence of abnormal circulating apoA-I variants $(21,22)$. Therefore, it is important to examine the prevalence of premature CHD in kindreds with isolated apoA-I deficiency. Two such kindreds have been identified $(20,23)$. Unfortunately, the information that can be learned from these is limited because only one member had xanthomatas and premature CHD in one kindred (20) and in the other kindred, the proband was only 5 yr old (23).

In this paper, we report a kindred with an isolated apoA-I deficiency caused by a structural mutation in the apoA-I gene, with five adult homozygotes. This allowed us to examine the clinical and biochemical sequelae of an isolated apoA-I deficiency in one of the largest affected kindreds yet studied. 


\section{Methods}

\section{Subjects}

Proband. The proband was a 34-yr-old Caucasian woman who was ascertained through the Lipid Clinic at St. Michael's Hospital in June 1992 with undetectable plasma HDL. She was the product of a consanguineous marriage. She initially presented for medical attention at the age of $30 \mathrm{yr}$ with xanthelasma. In the same year, the patient was diagnosed to have bilateral cataracts requiring cataract extraction on the right eye. She also had bilateral subretinal lipid deposition with exudative proliferative retinopathy complicated by bilateral retinal detachments, which were treated surgically. She had a long-standing history of mild imbalance. She had no history of diabetes mellitus or hypertension, or any symptom suggestive of coronary or vascular insufficiency. Other history of past health included tonsillectomy at age $13 \mathrm{yr}$. She smoked one pack of cigarettes a day for $20 \mathrm{yr}$. On physical examination, she had a body mass index of $25.4 \mathrm{~kg} / \mathrm{m}^{2}$, bilateral punctate xanthelasmata of the upper eyelids, mildly thickened Achilles tendons, mild midline cerebellar ataxia, and asymmetric bilateral neurosensory hearing loss.

Initial biochemical evaluation before referral included determination of plasma lipid and lipoprotein as follows: total cholesterol was $6.88 \mathrm{mM}$, triglycerides were $2.87 \mathrm{mM}$, HDL-cholesterol (HDL-C) was $0.06 \mathrm{mM}$, and LDL-cholesterol (LDL-C) was $5.76 \mathrm{mM}$. She was initiated on gemfibrozil but was switched to lovastatin because of lack of efficacy. Fasting glucose, glycosylated hemoglobin, serum electrolytes, liver function tests, serum protein electrophoresis, and thyroid function tests were all normal. Both resting electrocardiogram and stage 5 Bruce protocol stress electrocardiogram were normal. Computed tomography scan of the head had normal results. Audiometry showed mild bilateral asymmetrical neurosensory hearing loss.

First-degree relatives. The proband's father died suddenly due to a myocardial infarction at the age of $64 \mathrm{yr}$ with no antecedent ischemic symptoms. Her mother died of stomach cancer at age 51 . Nine of the proband's eleven siblings were studied biochemically. One sister (II-c, Fig. 1), aged $38 \mathrm{yr}$, suffered a myocardial infarction at age 34 . She underwent two-vessel coronary bypass surgery $3 \mathrm{yr}$ later. Other risk factors include a 25 pack-year smoking history and "hypercholesterolemia" for several years. She had bilateral xanthelasmata, Achilles tendon xanthomata, and planar xanthomata in the web spaces of the hands, cubital and popliteal fossae. She also had a mild degree of cerebellar ataxia. Her tonsils were normal. A second sister (II-b, Fig. 1), aged $39 \mathrm{yr}$, had hypertension, obesity, "hyperlipidemia" for several years and angina with documented reversible myocardial ischemia on thallium stress test. She also had cerebellar ataxia. The remaining sibs, aged from 24 to $41 \mathrm{yr}$, were asymptomatic for CHD, neuropathy, or visual impairment.

\section{Lipid analyses}

Plasma lipids and lipoproteins. The plasma lipid and lipoprotein profiles were determined in the J. A. Little Lipid Research Laboratory using the Lipid Research Clinics' protocol (30). Blood samples were obtained from subjects after a 12-14-h fast and collected into tubes containing $\mathrm{Na}_{2}$-EDTA. Plasma was separated after centrifugation at $3,000 \mathrm{rpm}$ at $4^{\circ} \mathrm{C}$ for $30 \mathrm{~min}$. VLDL was isolated by ultracentrifugation of plasma at $45,000 \mathrm{rpm}$ for $16.5 \mathrm{~h}$ at $10^{\circ} \mathrm{C}$ using a model $50.3 \mathrm{Ti}$ rotor in an L8-80 centrifuge (Beckman Instruments, Inc., Fullerton, CA). HDL was isolated from the infranatant with dextran sulfate precipitation. HDL $(d=1.063-1.21 \mathrm{~g} / \mathrm{ml}), \mathrm{HDL}_{2}(d=1.063-1.125 \mathrm{~g} /$ $\mathrm{ml})$, and $\mathrm{HDL}_{3}(d=1.125-1.21 \mathrm{~g} / \mathrm{ml})$ were prepared by sequential ultracentrifugation at $45,000 \mathrm{rpm}$ for $40 \mathrm{~h}$ at $10^{\circ} \mathrm{C}$. The total plasma and lipoprotein cholesterol and triglycerides were measured by automated enzymatic procedures using the model RA1000 ( Technicon Instruments, Tarrytown, NY). Free cholesterol and phospholipid were determined by gas chromatography (31).

Apolipoproteins and $\mathrm{Lp}(a)$. Apolipoprotein AI, AII, B, CII, CIII and $\mathrm{E}$ were measured using ELISA techniques $(32,33)$. Apo AI and

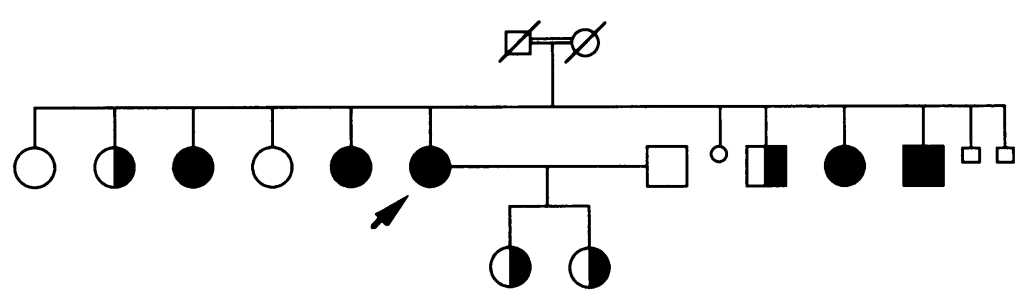

\begin{tabular}{|c|c|c|c|c|c|c|c|c|c|c|c|c|}
\hline IDENTIFICATION & $\| 1-\mathbf{a}$ & II-b & $\|-c$ & $\|-d$ & II-e & II-f & III-a & $I I I-b$ & $\|-f-s\|-g$ & II-h & II-i & $\|-j\|-k \|-1$ \\
\hline $\begin{array}{l}\text { Age } \\
\text { Total cholesterol (mM) } \\
\text { Triglyceride (mM) } \\
\text { HDL-cholesterol (mM) } \\
\text { LDL-cholesterol (mM) } \\
\text { VLDL-cholesterol (mM) } \\
\text { VLDL-C/TG ratio } \\
\text { free/total cholesterol }\end{array}$ & $\begin{array}{l}41 \\
4.85 \\
1.01 \\
0.81 \\
3.68 \\
0.36 \\
0.16 \\
0.28\end{array}$ & $\begin{array}{l}39 \\
6.06 \\
2.09 \\
0.56 \\
5.02 \\
0.48 \\
0.10 \\
0.31\end{array}$ & $\begin{array}{l}38 \\
5.43 \\
1.10 \\
0.10 \\
5.18 \\
0.15 \\
0.06 \\
0.32\end{array}$ & $\begin{array}{l}37 \\
6.34 \\
3.61 \\
0.80 \\
4.39 \\
1.15 \\
0.14 \\
0.27\end{array}$ & $\begin{array}{l}35 \\
5.11 \\
1.22 \\
0.08 \\
4.70 \\
0.33 \\
0.12 \\
0.30\end{array}$ & $\begin{array}{l}34 \\
5.77 \\
2.53 \\
0.08 \\
5.00 \\
0.69 \\
0.12 \\
0.33\end{array}$ & $\begin{array}{l}16 \\
4.47 \\
1.94 \\
0.55 \\
3.39 \\
0.53 \\
0.12 \\
0.28\end{array}$ & $\begin{array}{l}14 \\
3.33 \\
0.73 \\
0.57 \\
2.58 \\
0.18 \\
0.11 \\
0.26\end{array}$ & $\begin{array}{l}39 \\
3.81 \\
2.04 \\
0.86 \\
2.41 \\
0.54 \\
0.12 \\
0.31\end{array}$ & $\begin{array}{l}32 \\
5.09 \\
1.46 \\
0.72 \\
3.88 \\
0.49 \\
0.15 \\
0.27\end{array}$ & $\begin{array}{l}28 \\
4.64 \\
1.45 \\
0.09 \\
3.89 \\
\text { ND } \\
\text { ND } \\
\text { ND }\end{array}$ & $\begin{array}{l}26 \\
4.23 \\
1.81 \\
0.10 \\
3.78 \\
0.35 \\
0.08 \\
0.33\end{array}$ \\
\hline $\begin{array}{l}\text { apoA-I (g/) } \\
\text { apoB }(g / \mathrm{h}) \\
\text { apoA-II }(g / \mathrm{L}) \\
\text { apoC-II }(\mu \mathrm{g} / \mathrm{mL}) \\
\text { apoC-III }(\mu \mathrm{g} / \mathrm{mL}) \\
\text { apoE }(\mu \mathrm{g} / \mathrm{mL})\end{array}$ & $\begin{array}{l}1.06 \\
1.21 \\
0.21 \\
16.2 \\
68.9 \\
49.3\end{array}$ & $\begin{array}{l}0.70 \\
1.29 \\
0.17 \\
23.5 \\
73.4 \\
57.2\end{array}$ & $\begin{array}{l}<0.05 \\
1.49 \\
0.13 \\
19.5 \\
46.4 \\
64.9\end{array}$ & $\begin{array}{l}1.15 \\
1.72 \\
0.23 \\
42.1 \\
166.2 \\
152.8\end{array}$ & $\begin{array}{l}<0.05 \\
1.53 \\
0.07 \\
16.6 \\
47.9 \\
39.1\end{array}$ & $\begin{array}{l}<0.05 \\
1.77 \\
0.07 \\
32.1 \\
71.0 \\
74.9\end{array}$ & $\begin{array}{l}0.72 \\
1.15 \\
0.18 \\
32.4 \\
105.5 \\
46.1\end{array}$ & $\begin{array}{l}0.61 \\
0.81 \\
0.21 \\
17.4 \\
47.6 \\
32.0\end{array}$ & $\begin{array}{l}1.14 \\
0.92 \\
0.27 \\
28.3 \\
105.1 \\
35.8\end{array}$ & $\begin{array}{l}0.86 \\
1.30 \\
0.24 \\
32.9 \\
98.5 \\
48.2\end{array}$ & $\begin{array}{l}<0.05 \\
1.40 \\
0.09 \\
12.3 \\
40.4 \\
44.1\end{array}$ & $\begin{array}{l}<0.05 \\
1.21 \\
0.08 \\
10.4 \\
41.9 \\
33.0\end{array}$ \\
\hline apoE is & $4 / 3$ & $3 / 3$ & $3 / 3$ & $4 / 4$ & $3 / 3$ & $3 / 3$ & $3 / 3$ & $3 / 3$ & $3 / 3$ & $3 / 3$ & $4 / 4$ & $4 / 3$ \\
\hline apoA-I genotype & AA & $\mathrm{Aa}$ & aa & AA & aa & aa & $\mathrm{Aa}$ & $\mathrm{Aa}$ & $A A$ & $\mathrm{Aa}$ & aa & aa \\
\hline
\end{tabular}

Figure 1. Biochemical features in subjects from pedigree AI Q[-2]X. Proband is indicated by the arrow. Small symbols indicate subjects not sampled. ND indicates analysis not done. Plasma apoA-I concentrations shown were determined nephelometrically. The proband's plasma apoA-I concentration determined by ELISA was $<0.01 \mathrm{~g} /$ liter. ApoA-I genotypes shown indicate the presence of either homozygosity for the wildtype allele (AA), heterozygosity ( $\mathrm{Aa}$ ), or homozygosity for the AI Q [-2]X mutation allele (aa) as determined by Fnu4HI RFLP analysis. Reference values for plasma apolipoprotein levels, based on 87 normal control subjects and expressed as mean \pm SD, are apoA-I, $1.55 \pm 0.22 \mathrm{~g} /$ liter; apoB, $1.22 \pm 0.32 \mathrm{~g} /$ liter; apoA-II, $0.33 \pm 0.06 \mathrm{~g} /$ liter; apoCII, $29 \pm 15 \mu \mathrm{g} / \mathrm{ml}$; apoCIII, $116 \pm 44 \mu \mathrm{g} / \mathrm{ml}$; and apoE, $50 \pm 21 \mu \mathrm{g} / \mathrm{ml}$. 
apo B levels were also measured by nephelometry (Behring, Montreal, Canada). Plasma Lp (a) was determined by ELISA (Terumo Medical Corp., Elkton, MD).

\section{Electrophoretic procedures}

Agarose gel electrophoresis, SDS-glycerol electrophoresis, isoelectric focusing, and immunoblotting procedures were performed as previously described (34-36). HDL particle sizes were determined by nondenaturing gradient PAGE using precast 4-30\% gels (Pharmacia LKB Biotechnology, Uppsala, Sweden) (37). In order to visualize the particles, aliquots corresponding to $10-20 \mu \mathrm{g}$ of $\mathrm{HDL}$ protein were applied to the gel. Well sizes were adjusted to accommodate the larger volumes of HDL required for the subjects with severe HDL deficiency. Proteins were stained with $0.025 \%$ Coomassie Brilliant Blue G-250 (Eastman Kodak Co., Rochester, NY), and the gels were scanned with a densitometer (Helena Laboratories, Beaumont, TX). The gels were calibrated with a standard protein mixture (HMW Calibration Kit, Pharmacia LKB Biotechnology) consisting of thyroglobulin (hydrated particle size $17.0 \mathrm{~nm})$, ferritin $(12.2 \mathrm{~nm})$, lactate dehydrogenase $(8.16$ $\mathrm{nm})$, and bovine serum albumin $(7.1 \mathrm{~nm})$. Migration distances of peaks were measured relative to bovine serum albumin. Particle sizes of HDL fractions were calculated from the calibration curve of peak migration distance values against hydrated particle size of the standard protein mixture with nonlinear regression curve fitting software (GraphPad Software, San Diego, CA).

Lp-AI particles in plasma, $\mathrm{HDL}, \mathrm{HDL}_{2}$, and $\mathrm{HDL}_{3}$ subfractions were measured by differential electroimmunodiffusion assay (Sebia, Issy-les-Moulineaux, France) (38).

\section{DNA analyses}

PCR amplification. Genomic DNA was prepared from the proband and 11 first-degree relatives. Based on genomic sequence information $(39,40)$, we designed oligonucleotide primers (Table I) to amplify genomic regions spanning exons 2,3 , and 4 of the apoA-I gene using the Taq PCR. Reactions were carried out in a $100-\mu l$ volume and contained $1 \mu \mathrm{g}$ of DNA, $200 \mu \mathrm{M}$ of dNTPs, $0.1 \mathrm{mM}$ of primers, and $2.5 \mathrm{U}$ of Taq polymerase (Gibco BRL, Gaithersburg, MD). Cycling was performed with a Biosycler oven (BIOS Corp., New Haven, CT) as follows: DNA was initially denatured at $94^{\circ} \mathrm{C}$ for $10 \mathrm{~min}$ followed by the addition of the reaction mixture and Taq polymerase and three cycles of $93^{\circ} \mathrm{C}(1 \mathrm{~min}), 60^{\circ} \mathrm{C}(30 \mathrm{~s})$, and $72^{\circ} \mathrm{C}(30 \mathrm{~s})$ followed by 30 cycles of $93^{\circ} \mathrm{C}(20 \mathrm{~s}), 60^{\circ} \mathrm{C}(20 \mathrm{~s})$, and $72^{\circ} \mathrm{C}(30 \mathrm{~s})$ incubation.

Cloning and sequencing of the PCR-amplified DNA. The PCR product was purified by extraction with phenol followed by precipitation with 7.5 $\mathrm{M} \mathrm{NH}_{4} \mathrm{OAc}$ and absolute alcohol. The purified DNA was then sequentially digested with BamHI and EcoRI (New England Biolabs, Beverly, MA), gel purified, ligated into pUC 19 , and subcloned into $E$. coli $\mathrm{DH} 5 \alpha$ as previously described (41). Sequencing of the denatured

Table I. Sequence and Position of Oligonucleotide Primers

\begin{tabular}{lrr}
\hline Primer & Sequence & Position* $^{*}$ \\
\hline 1. & 5'-ATAGAATTCTTCTGCATGCTGAAGGCA-3' & 634 \\
2. & 5'-ATAGGATCCGCCAGTGAGAAACCTGCT-3' & 845 \\
3. & 5'-ATAGAATTCAGCAGGTTTCTCACTGGC-3' & 828 \\
4. & 5'-ATAGGATCCAGTCTGGCTTCAACATCA-3' & 1144 \\
5. & 5'-ATAGAATTCTACTGGAAATGCTAGGCC-3' & 1565 \\
6. & 5'-ATAGGATCCTTCTCCTTGAGAGCCTCA-3' & 2100 \\
7. & 5'-ATAGAATTCAAGCTGCACGAGCTGCAA-3' & 1946 \\
8. & 5'-ATAGGATCCA_TTCTGAGCACCGGGAAG-3' & 2318 \\
\hline
\end{tabular}

* The position number is that of the nucleotide underlined, which corresponds to the $5^{\prime}$ end of the genomic part of the primer. The numbering system is according to Shoulders et al. (40). Each primer includes a $5^{\prime}$ nongenomic 9-mer sequence for ligation. double-stranded DNA was carried out using T7 polymerase (United States Biochemicals, Cleveland, $\mathrm{OH}$ ) according to the supplier's protocol.

Apo A-I genotyping. PCR-amplified exon 3 of the apoA-I gene with primers 3 and 4 from the proband and 11 family members were digested with BbvI or Fnu4HI (New England Biolabs) for $4 \mathrm{~h}$. The digestion conditions were those recommended by the supplier. The digestion reaction was then electrophoresed on a $4 \%$ polyacrylamide gel, stained with ethidium bromide, and subsequently visualized under UV light. The PCR-amplified exon 3 from a series of 75 normolipidemic controls were genotyped with Fnu4HI digestion in a similar manner.

Apo E genotyping. Apo $\mathrm{E}$ isotyping was determined for the proband and 11 family members using Hhal digestion of PCR amplified apoE gene (42).

\section{Statistical analysis}

Correlation of plasma apoA-I with apoA-II was performed with standard linear regression.

\section{Results}

Plasma lipids and apolipoproteins. Concentrations of plasma lipids and apolipoproteins for members of this kindred are presented in Fig. 1. The proband and four of her 11 siblings (II-c, $e, i, j$ ) had severe deficiency of HDL ( HDL $<0.1 \mathrm{mM}$ ) and nondetectable plasma apoA-I. Agarose gel electrophoresis of these subjects showed absence of the $\alpha$-migrating band, establishing the biochemical diagnosis of AAL. IEF and immunoblotting of the proband's plasma also showed no evidence of any apoA-I isoform (data not shown). Two siblings (II-b, h) and both of the proband's two daughters (III-a, b) had reduced plasma HDL to $41-52 \%$ of age- and sex-matched median. Their plasma apoA-I levels were $0.61-0.86 \mathrm{~g} /$ liter. Two other sibs and the proband's husband had reduced plasma HDL (56$58 \%$ of median for age and sex ) but near normal plasma apoA-I levels (1.06-1.15 g/liter).

The proband, despite ongoing lovastatin therapy, had total cholesterol exceeding the 90th percentile and LDL-cholesterol exceeding the 95th percentile, and triglyceride exceeding the 95 th percentile. She also had elevated plasma apoB, markedly reduced but detectable apoA-II and normal apoC-III.

Plasma LDL-cholesterol levels exceeded the 90th percentile in three of the four siblings (II-c, e, j) with AAL. Two of the remaining sibs (II-b, d) and one daughter (III-a) also had LDL$\mathrm{C}$ exceeding the 90 th percentile. Triglyceride levels were normal in the four siblings with AAL but exceeding the 90th percentile in the two sibs (II-b, d) and one of the two daughters (III-a). $L p(a)$ levels of the members ranged from 0.8 to 7.8 $\mathrm{mg} / \mathrm{dl}$ and were within the normal range.

Plasma apoA-II levels also varied significantly among the family members. Subjects with AAL had markedly reduced but detectable plasma apoA-II. The remaining subjects had moderately reduced plasma apoA-II and the plasma apoA-II levels correlated positively with plasma apoA-I levels $(r=0.87$; slope $=0.158$ with $95 \%$ confidence interval: $-0.001-0.317)$.

Mean plasma level of apoC-III was $49.5 \mu \mathrm{g} / \mathrm{ml}(\mathrm{SD}=12.4$ $\mu \mathrm{g} / \mathrm{ml}$ ) for subjects with AAL, $81.2 \mu \mathrm{g} / \mathrm{ml}(\mathrm{SD}=26.33 \mu \mathrm{g} /$ $\mathrm{ml}$ ) for members with intermediate plasma apoA-I (IIb, h; III-a, b), and $113.4 \mu \mathrm{g} / \mathrm{ml}(\mathrm{SD}=49.2 \mu \mathrm{g} / \mathrm{ml})$ for those with near normal plasma apoA-I (II-a, d; II-f-s). The ratio of free cholesterol/total cholesterol ranged from 0.30 to 0.33 in AAL subjects and from 0.26 to 0.28 in the remaining members.

DNA analysis. Sequencing of three separate clones showed a single $\mathrm{C} \rightarrow \mathrm{T}$ substitution at codon $[-2]$ of the apoA-I gene in 


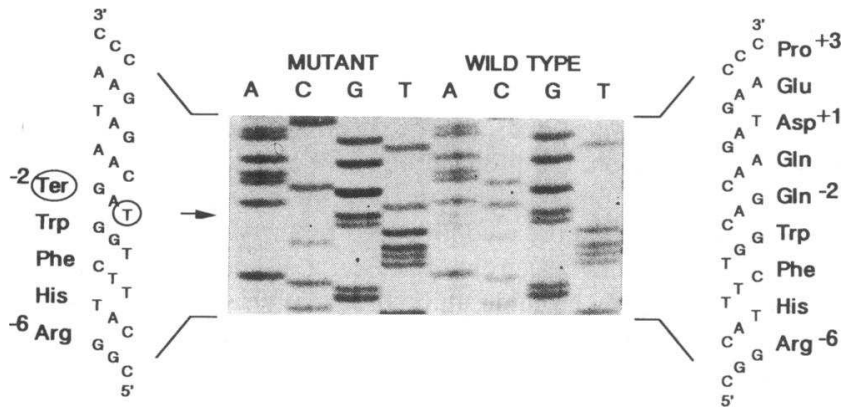

Figure 2. ApoA-I Q[-2]X DNA sequence. The autoradiogram shows the nucleotide change from $C$ to $T$ at codon -2 , which resulted in the change of a codon encoding for the amino acid glutamine $(Q)$ to a premature termination codon $(\mathrm{X})$.

exon 3 (Fig. 2). This mutation was detected in all three clones sequenced which suggested homozygosity. The mutation, designated $\mathrm{Q}[-2] \mathrm{X}$, transforms a codon at position [ -2$]$ relative to the first amino acid of circulating mature apoA-I. The normal sequence at this position encodes glutamine, but the mutated codon encodes premature termination. Cloned sequences of exons 2 and 4 of the proband's DNA were normal. Endonuclease digestions of the proband's DNA exon 3 fragment with $\mathrm{BbvI}$ and Fnu4HI each showed complete loss of a digestion site as predicted by the mutant sequence, confirming the mutation and the homozygosity.

ApoA-I genotypes using Fnu4HI were determined in the family members (Fig. 3 ) and in a series of 75 normolipidemic Caucasian controls. All 75 control subjects showed homozygosity for the apoA-I wild isotype. All AAL subjects showed homozygosity for the apoA-I Q [-2]X allele. The proband's husband and two sibs (II-a, d) showed homozygosity for wild type apoAI. The proband's two obligate heterozygote daughters (III-a, b), and two other sibs (II-b, h) showed heterozygosity for the wildtype and mutant alleles.

Gradient gel electrophoresis. Gradient gel electrophoresis of the homozygotes is illustrated in Fig. 4. The HDL fraction is dominated by a population of very small particles with peak diameter of $7.9 \mathrm{~nm}$. In the heterozygotes, in addition to the particles of size $7.9 \mathrm{~nm}$ in diameter, a second population with peak diameter of $8.4 \mathrm{~nm}$ can also be seen, which closely approximate the sizes of $\mathrm{HDL}_{3}$ subfractions seen in normal sub- jects. In heterozygotes there is no HDL seen with peak size in the $\mathrm{HDL}_{2}$ range.

Differential electroimmunodiffusion. Lp-AI particles in whole plasma, $\mathrm{HDL}, \mathrm{HDL}_{2}$, and $\mathrm{HDL}_{3}$ subfractions were separated by differential electroimmunodiffusion. Lp-AI particles were detected in the plasma, $\mathrm{HDL}, \mathrm{HDL}_{2}$, and $\mathrm{HDL}_{3}$ fractions among the unaffected members (data not shown). For the heterozygotes, the same particles were detected in plasma, HDL, $\mathrm{HDL}_{3}$, but not the $\mathrm{HDL}_{2}$ subfractions (data not shown). For the homozygotes, Lp-AI was undetectable (data not shown).

\section{Discussion}

We have identified a Canadian kindred with a novel nonsense mutation of the apoA-I gene which we designate as Q[-2]X. Homozygosity for $\mathrm{Q}[-2] \mathrm{X}$ causes a selective failure to produce any portion of the mature apoA-I. This results in nondetectable plasma apoA-I and a very low level of HDL-C. Heterozygosity for $\mathrm{Q}[-2] \mathrm{X}$ results in intermediate levels of apoA-I and HDL-C, and the unaffected members have the highest HDL-C and apoA-I levels. This suggests the genetic trait is being transmitted in an autosomal co-dominant fashion. This mutation distinguishes itself from previously reported cases of genetically determined HDL deficiencies. Of the nine siblings ascertained, five were homozygous for the mutation. This is the largest number of adult homozygotes in any single kindred with apoA-I deficiency. There is a high prevalence of premature CHD among affected subjects.

The clinical manifestation appears highly heterogeneous among the homozygotes. The proband had clinical findings of cerebellar neuropathy, bilateral premature cataracts, subretinal lipid deposition complicated by retinal detachment, and tendon xanthomata. The first two of these signs have not been previously described in any genetically determined HDL deficiency. Their relevance to the apoA-I mutation in this family remains to be clarified. Retinopathy related to subretinal lipid deposition observed in the proband is unique among kindreds with genetically determined apoA-I deficiency. Retinal involvement has only been reported in two cases of patients with lecithin:cholesterol acyltransferase LCAT deficiency (43). The clinical manifestations of one homozygote sister (II-c) included arcus cornealis, planar xanthomata, and premature CHD. Although planar xanthoma and/or corneal abnormalities appeared to distinguish subjects with severe HDL defi-
A

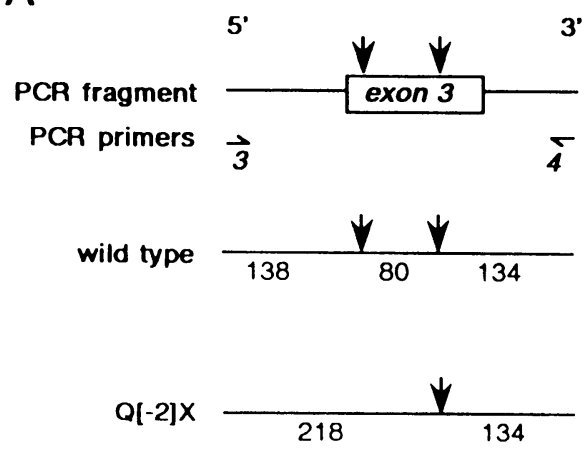

B

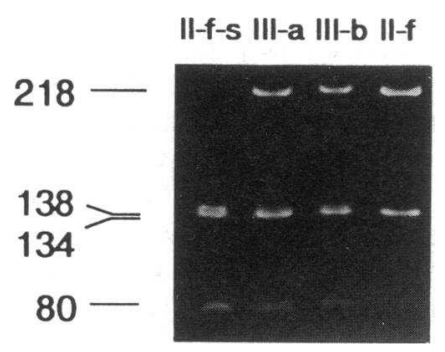

Figure 3. (A) Restriction map for exon 3 . The position of Fnu4HI restriction sites are indicated with vertical arrows. Fnu4HI fragment sizes (in bp) are shown for alleles containing the normal apoA-I gene exon 3 sequence ("wild type") and the mutant apoA-I gene exon 3 sequence ("Q $[-2] X ")$. The regions of the genomic DNA sequence corresponding to PCR primers 3 and 4 are indicated. $(B)$ Segregation of Fnu4HI

in a partial $\mathrm{Q}[-2] \mathrm{X}$ pedigree. The unaffected member (II-f-s), the proband's husband showed the wild-type Fnu4HI RFLP. The $\mathrm{Q}[-2] \mathrm{X}$ allele obliterates a Fnu4HI site, creating a larger band ( $218 \mathrm{bp})$ in the proband (II-f). The two daughters, who are obligate heterozygotes, showed combined wild-type and Q[-2]X Fnu4HI RFLP. 


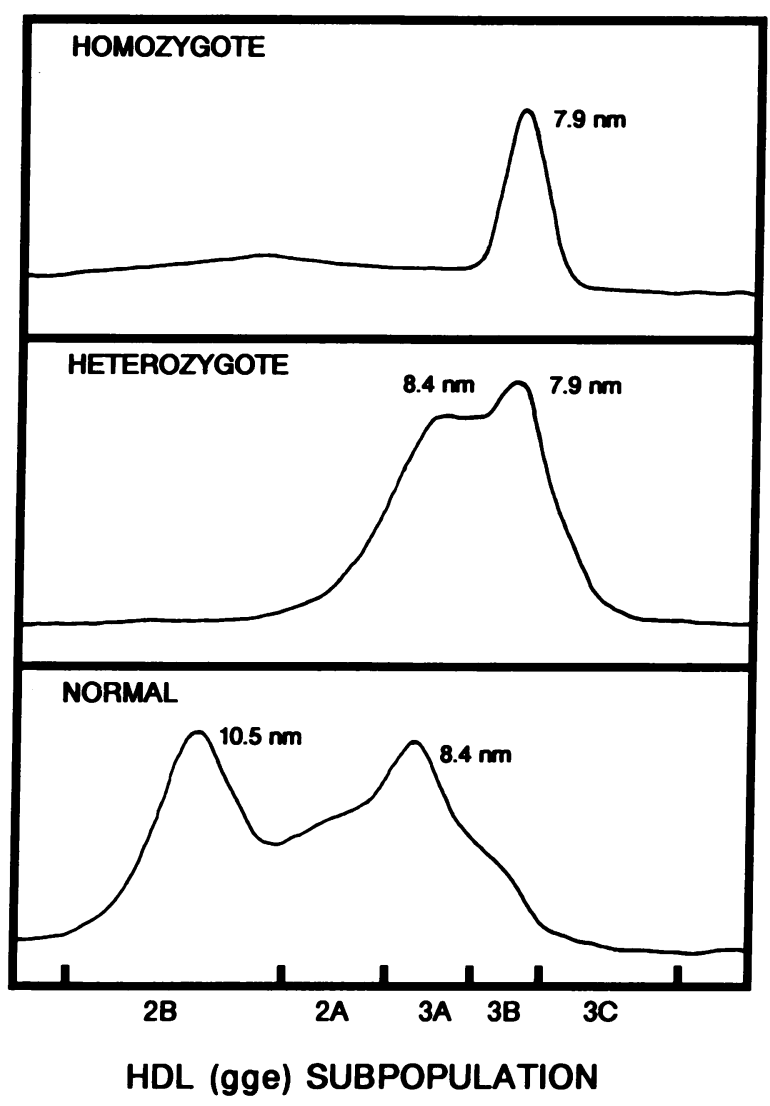

Figure 4. HDL particle size profiles. Densitometric scans after nondenaturing 4-30\% PAGE of HDL isolated from an AI Q[-2]X homozygote (top), heterozygote (center), and a normal subject (bottom). HDL subpopulation intervals are shown below the scan profile and peak particle diameters are given in nanometer.

ciency among a number of other kindreds reported (15-23, 26), this appears not to be the case in our kindred. All of the remaining homozygotes, though not available for detailed clinical examination, were contacted and had no complaints related to CHD, neuropathy, or visual impairment. Among the heterozygotes, one sister (II-b) had reversible CHD on stress thallium testing but this was confounded by other CHD risk factors including cigarette smoking, hypertension, and obesity. She also complained of mild imbalance. The remaining heterozygotes and unaffected siblings had no complaints related to the above illnesses.

The affected members of the kindred not only had a reduced number of HDL particles but also an abnormal HDL size distribution as shown by the gradient gel electrophoresis. The HDL among homozygotes was dominated by a fraction of very small HDL particles with peak diameter of $7.9 \mathrm{~nm}$. The heterozygotes had two populations of HDL particles, one with peak diameter of $7.9 \mathrm{~nm}$ and the other with peak diameter of $8.4 \mathrm{~nm}$, resembling $\mathrm{HDL}_{3}$ particles in normal individuals. On the other hand, the heterozygotes were virtually devoid of any HDL particles in the $\mathrm{HDL}_{2}$ flotation range. Among the unaffected relatives, the HDL particle distribution appeared normal.

Differential electroimmunodiffusion assay of the HDL fractions of the homozygotes showed the presence of apoA-II but not apoA-I, which suggests that the small HDL particles seen in gradient gel electrophoresis were Lp-AII. Detection of Lp-AII particles have been described in several reports of apoA-I deficiencies $(22,26,28)$. In transgenic mice overexpressing apoA-II (44), human apoA-II-only particles of $8 \mathrm{~nm}$ diameter were observed. Taken together these observations further support the stable formation of human apoA-II-only particle of a distinct size when apoA-II is unable to interact with human apoA-I due either to the absence of apoA-I or structural abnormalities of apoA-I, or in human apoA-II transgenic mice containing only murine apoA-I. These observations also reinforce the concept that apoA-I is the major determinant of HDL structure as well as plasma level.

The plasma levels of apoC-III were only moderately reduced in the AAL subjects which suggests absence of a gross functional defect of the apoC-III gene associated with the apoA-I mutation. The reduction in plasma apoC-III is presumably due to a lack of circulating HDL particles to accept transfer of apoC-III from the triglyceride-rich particles, resulting in increased catabolism. This further confirms that the present mutation results in a selective failure to produce any portion of the mature apoA-I. The free cholesterol/total cholesterol ratio was normal for both affected and unaffected members. This is in agreement with previous findings that apoA-I is not essential for LCAT activity (45).

The LDL-C in 7 of 11 members in the kindred exceed the 90th percentile after adjustment for age and gender. Four of these seven subjects have concurrent elevation of triglycerides over the 90th percentile (type IIb). Such findings are not uniform among reported cases of genetically determined HDL deficiency and are unlikely to be pathogenetically related to the apoA-I deficiency. The cause of the hyperlipidemia remains to be elucidated.

The severe HDL deficiency clearly predisposes some of the young members in this kindred to premature CHD. One of the five homozygotes (II-c) suffered a myocardial infarction due to coronary atherosclerosis at age 34 , whereas the other homozygote sibs so far had no signs of CHD. One heterozygote (II-b) had documented reversible myocardial ischemia which was confounded by the presence of numerous coronary risk factors including elevated LDL-C, hypertension, cigarette smoking, and obesity. The father, an obligate heterozygote, died of massive $\mathrm{MI}$ at the age of $64 \mathrm{yr}$ with no antecedent symptoms. The incidence of CHD among the homozygotes is lower than that of homozygous familial hypercholesterolemia (46). There is no history of early CHD related mortality in this kindred and the homozygous subjects have all lived to their reproductive age. These observations appear to parallel the lack of fulminant atherosclerosis during the early stage of development in mice after apoA-I deletion by homologous recombination (47) although there are fundamental differences in the major lipoprotein classes between mice and human that make the direct comparisons difficult.

It appears that inability to synthesize apoA-I or assemble HDL particles may be the common denominator among the kindreds predisposed to CHD. Two kindreds with combined deficiency of apoA-I and apoC-III, one due to apoA-I and apoC-III gene inversion defect $(15,16)$ and the other due to deletion of the entire apoA-I-CIII-AIV gene complex $(17,18)$, rendering the individuals unable to synthesize apoA-I and apoC-III in the former case and additional apoA-IV synthetic defect in the latter case, appeared most susceptible to premature CHD. A Japanese family with an apoA-I gene codon 84 
nonsense mutation $(19,20)$ and a presumed HDL assembly defect also appeared to be susceptible. Our kindred, with an isolated apoA-I synthetic defect also confirms this association with CHD. The opposite was true for subjects with homozygous apoA-I $\mathrm{I}_{202: \mathrm{fs}}(21)$, heterozygous apoA-I $\mathrm{I}_{\text {Seatle }}(22)$, and apoA-I Iowa $(48)$ defects, all of which manifest circulating apoAI variants. Clinically, the former also had added planar xanthoma, but the latter three had arcus cornealis, corneal clouding and systemic amyloidosis respectively. Furthermore, patients with Tangier disease (29) have unique abnormal deposition of lipids and hypercatabolism of apoA-I and are spared from increased risk of CHD. LCAT deficiency causes severe HDL deficiency and premature atherosclerosis in addition to hemolytic anemia, corneal deposit and glomerulopathy (25). A variety of structural defects of the LCAT gene have been reported $(49,50)$. Fish eye disease $(51,52)$ patients also have severe HDL deficiency, putatively due to specific loss of LCAT-HDL interaction as a result of specific structural mutations of the LCAT gene. Fish-eye disease subjects are spared from premature CHD. It is probable that specific lipid-tissue interaction plays a major role in modulating an individual's susceptibility to CHD.

In summary, we have reported a large Canadian kindred with a genetic mutation $\mathrm{Q}[-2] \mathrm{X}$ in the apoA-I gene causing specific failure of production of apoA-I resulting in AAL. Clinically, the five homozygotes had uniformly low HDL levels but were highly heterogeneous with regard to clinical features. The retinal lipid deposits described in the proband are unique. There was a marked increase in prevalence of premature CHD in this kindred, attributable to the unique $\mathrm{Q}[-2] \mathrm{X}$ mutation. The large size of this kindred, the diversity of clinical manifestations, and the established molecular defect of isolated absent apoA-I, should facilitate the search for critical determinants responsible for the diverse clinical phenotypes, with particular reference to the development of premature CHD.

\section{Acknowledgments}

We are indebted to Graham Maguire and Liling Tu for excellent technical assistance, and to Dr. Glen Martin, Dr. Clive Mortimer, Dr. Bryan Temple, and Dr. Wendy Rosenthall for their contributions in clinical evaluations of the subjects. We also thank Klara Geher and Dr. Arnis Kuksis for measurements of free cholesterol.

These studies were supported in part by the Medical Research Council of Canada, Heart and Stroke Foundations of Canada and Ontario. D. S. Ng is a postdoctoral fellow supported by the Banting and Best Diabetes Centre, University of Toronto, Canada. R. A. Hegele is a McDonald Scholar of the Heart and Stroke Foundation of Canada.

\section{References}

1. Castelli, W. P. 1988. Cholesterol and lipids in the risk of coronary artery disease: the Framingham Heart Study. Can. J. Cardiol. 4(Suppl. A):5A-11A.

2. Brunner, D., J. Weisbort, N. Meshulam, S. Schwartz, J. Gross, H. SaltzRennert, S. Altman, and K. Loebl. 1987. Relationship of serum total cholesterol and high density lipoprotein cholesterol percentage to the incidence of definite coronary events: twenty year follow up of Donolo-Tel Aviv prospective coronary artery disease study. Am. J. Cardiol. 59:1271-1276.

3. Gordon, D. J., J. L. Probstfield, R. J. Garrison, J. D. Neaton, W. P. Castelli, J. D. Knoke, D. R. Jacobs, Jr., S. Bangdiwala, and H. A. Taylor. 1989. High-density lipoprotein cholesterol and cardiovascular disease: four prospective American studies. Circulation. 79:8-15.

4. Assmann, G., H. Schulte, H. Funke, A. von Eckardstein, and U. Seedorf. 1989. The prospective cardiovascular Munster (PROCAM) study: identification of high-risk individuals for myocardial infarction and the role of high-density lipoprotein. In High Density Lipoproteins and Atherosclerosis II. N. E. Miller, editor. Excerpta Med. Int. Congr. Ser. 826:51-65.

5. Manninen, V., L. Tenkanen, P. Koskinen, J. K. Huttunen, M. Măntäri, O. P. Heinonen, and M. H. Frick. 1992. Joint effects of serum triglyceride and LDL cholesterol and HDL cholesterol concentrations on coronary heart disease risk in the Helsinki study. Circulation. 85:37-45.

6. Johnson, W. J., F. H. Mahlberg, G. H. Rothblat, and M. Phillips. 1991. Cholesterol transport between cells and high-density lipoproteins. Biochim. Biophys. Acta. 1085:273-298.

7. Miller, N. E. 1987. Associations of high-density lipoprotein subclasses and apolipoproteins with ischemic heart disease and coronary atherosclerosis. $\mathrm{Am}$. Heart. J. 113:589-597.

8. Stampfer, M. J., F. M. Sacks, S. Salvini, W. C. Willett, and C. H. Hennekens. 1991. A prospective study of cholesterol, apolipoproteins, and the risk of myocardial infarction. N. Engl. J. Med. 325:373-381.

9. Cheung, M. C., and J. J. Albers. 1984. Characterization of lipoprotein particles isolated by immunoaffinity chromatography: particles containing A-I and A-II and particles containing A-I but no A-II. J. Biol. Chem. 259:1220112209.

10. Barbaras, R., P. Puchois, J. C. Fruchart, and G. Ailhaud. 1987. Cholesterol efflux from cultured adipose cells is mediated by LpAI particles but not by LpAI:AII particles. Biochem. Biophys. Res. Commun. 142/1:63-69.

11. Johnson, W. J., E. P. C. Kilsdonk, A. van Tol, M. C. Phillips, and G. H. Rothblat. 1991. Cholesterol efflux from cells to immunopurified subfractions of human high density lipoprotein: LP-AI and LP-AI/AII. J. Lipid Res. 32:19932000 .

12. Kunitake, S. T., M. R. Jarvis, R. L. Hamilton, and J. Kane. 1991. Binding of transition metals by apolipoprotein A-I containing plasma lipoproteins: Inhibition of oxidation of low density lipoproteins. Proc. Natl. Acad. Sci. USA. 89:6993-6997.

13. Parthasarathy, S., J. Barnett, and L. G. Fong. 1990. High density lipoprotein inhibits the oxidative modification of low density lipoprotein. Biochim. Biophys. Acta. 1044:275-283.

14. Khoo, J. C., E. Miller, P. McLoughlin, and D. Steinberg. 1990. Prevention of low density lipoprotein aggregation by high density lipoprotein or apolipoprotein A-I. J. Lipid. Res. 31:645-652.

15. Norum, R. A., J. B. Lakier, S. Goldstein, A. Angel, R. B. Goldberg, W. D. Block, D. K. Noffze, P. J. Dolphin, J. Edelglass, D. D. Bogorad, and P. Alaupovic. 1982. Familial deficiency of apolipoproteins A-I and C-III and precocious coronary artery disease. N. Engl. J. Med. 306:1513-1519.

16. Karathanasis, S. K., E. Ferris, and E. A. Haddad. 1987. DNA inversion within the apolipoproteins AI/CIII/AIV-encoding gene cluster of certain patients with premature atherosclerosis. Proc. Natl. Acad. Sci. USA. 84:7198-7202.

17. Schaefer, E. J., J. M. Ordovas, S. W. Law, G. Ghiselli, M. L. Kashyap, L. S. Srivastava, W. H. Heaton, J. J. Albers, W. E. Connor, F. T. Lindgren, Y. Lemeshev, et al. 1985. Familial apolipoprotein A-I and C-III deficiency variant II. $J$. Lipid Res. 26:1089-1101.

18. Ordovas, J. M., D. K. Cassidy, F. Civeira, C. Bisgaier, and E. J. Schaefer. 1989. Familial apolipoprotein A-I, C-III, and A-IV deficiency and premature atherosclerosis due to deletion of a gene complex on chromosome $11 . \mathrm{J}$. Biol. Chem. 264:16339-16342.

19. Hiasa, Y., T. Maeda, and H. Mori. 1986. Deficiency of apolipoproteins A-I and C-III and severe coronary heart disease. Clin. Cardiol. 9:349-352.

20. Matsunaga, T., Y. Hiasa, H. Yanagi, T. Maeda, N. Hattori, K. Yamakawa, Y. Yamanouchi, I. Tanaka, T. Obara, and H. Hamaguchi. 1991. Apolipoprotein A-I deficiency due to a codon 84 nonsense mutation of the apolipoprotein A-I gene. Proc. Natl. Acad. Sci. USA. 88:2793-2797.

21. Funke, H., A. von Eckardstein, P. H. Pritchard, M. Karas, J. J. Albers, and G. Assmann. 1991. A frameshift mutation in the human apolipoprotein A-I gene causes high density lipoprotein deficiency, partial lecithin:cholesterol-acyl-transferase deficiency, and corneal opacities. J. Clin. Invest. 87:371-376.

22. Deeb, S. S., M. C. Cheung, R. Peng, A. C. Wolf, R. Stern, J. J. Albers, and R. H. Knopp. 1991. A mutation in the human apolipoprotein A-I gene. J. Biol. Chem. 266:13654-13660.

23. Schmitz, G., and K. Lackner. 1989. High density lipoprotein deficiency with xanthomas: a defect in apo A-I synthesis? In Atherosclerosis VII. G. Crepaldi, editor. Elsevier, Amsterdam. 399-403.

24. Carlson, L. A., and B. Philipson. 1979. Fish-eye disease. A new familial condition with massive corneal opacities and dyslipoproteinemia. Lancet. 8149:921-923.

25. Breslow, J. L. 1989. Familial disorders of high density lipoprotein metabolism. In The Metabolic Basis of Inherited Diseases. 6th edition. C. R. Scriver, A. L. Beaudet, W. S. Sly, and D. Valle, editors. McGraw-Hill, Inc., New York, 1251-1266.

26. Bekaert, E. D., P. Alaupovic, C. S. Knight-Gibson, M. J. Laux, J. M Pelachyk, and R. A. Norum. 1991. Characterization of apoA- and apoB-containing lipoprotein particles in a variant of familial apoA-I deficiency with planar xanthoma: the metabolic significance of LP-A-II particles. J. Lipid Res. 32:15871599. 
27. Gustafson, A., W. J. McConathy, P. Alaupovic, M. D. Curry, and B. Persson. 1979. Identification of lipoprotein families in a variant of human plasma apolipoprotein A deficiency. Scand. J. Clin. Lab. Invest. 39:377-387.

28. Cheung, M. C., A. J. Mendez, A. C. Wolf, and R. H. Knopp. 1993. Characterization of apolipoprotein A-I- and A-II-containing lipoproteins in a new case of high density lipoprotein deficiency resembling Tangier disease and their effects on intracellular cholesterol efflux. J. Clin. Invest. 91:522-529.

29. Assmann, G., G. Schmitz, and H. B. Brewer, Jr. 1989. Familial high density lipoprotein deficiency: Tangier disease. In The Metabolic Basis of Inherited Diseases. C. R. Scriver, A. L. Beaudet, W. S. Sly, and D. Valle. McGraw Hill, Inc., New York. 1267-1282.

30. Lipid Research Clinics Program. 1982. Lipid and lipoprotein analysis. In Manual of Laboratory Operation. U. S. Department of Health, Education and Welfare, Government Printing Office, Washington, D. C. Publication No. 75678.

31. Kuksis, A., J. J. Myher, L. Marai, and K. Geher. 1975. Determination of plasma lipid profiles by automated gas chromatography and computerized data analysis. J. Chromatogr. Sci. 13:423-430.

32. Voller, A., D. E. Bidwell, and A. Bartlet. 1979. Enzyme linked immunosorbent assay. Flowline Press, Guernsey, UK.

33. Bury, J., and M. Rosseneu. 1985. Quantitation of human serum apolipoprotein AI by enzyme immunoassay. Clin. Chem. 31:247-251.

34. Maguire, G. F., and W. C. Breckenridge. 1975. Agarose gel electrophoresis of plasma lipoprotein using the Durrum cell. Clin. Biochem. 8:161-168.

35. Maguire, G. F., M. Lee, and P. W. Connelly. 1989. Sodium dodecyl sulphate-glycerol polyacrylamide slab gel electrophoresis for the resolution of apolipoproteins. J. Lipid Res. 30:757-761.

36. Connelly, P. W., G. F. Maguire, T. Hofman, and J. A. Little. 1987. Structure of apolipoprotein $\mathrm{C}-\mathrm{II}_{\text {Toronto }}$, a nonfunctional human apolipoprotein. Proc. Natl. Acad. Sci. USA. 84:270-273.

37. Nichols, A. V., P. J. Blanche, and E. L. Gong. 1983. In Handbook of electrophoresis. L. Lewis, and J. Opplt, editors. CRC Press, Boca Raton, FL. 3:29.

38. Fruchart, J. C. 1990. Immunological determination of apoA-I containing lipoprotein particles. Atherosclerosis Rev. 20:233-238.

39. Karathanasis, S. K., V. I. Zannis, and J. L. Breslow. 1983. Isolation and characterization of the human apolipoprotein A-I gene. Proc. Natl. Acad. Sci. USA. 80:6147-6151.

40. Shoulders, C. C., A. R. Kornblihtt, B. S. Munro, and F. E. Baralle. 1983. Gene structure of human apolipoprotein AI. Nucleic Acid Res. 11:2827-2837.

41. Hegele, R. A., P. W. Connelly, G. F. Maguire, M. W. Huff, L. A. Leiter, B. M. Wolfe, A. J. Evans, and J. A. Little. 1991. An apolipoprotein CII mutation,
CII Lys19 $\rightarrow$ Thr identified in patients with hyperlipidemia. Dis. Markers. 9:7380.

42. Hixson, J. E., and D. T. Vernier. 1990. Restriction isotyping of apolipoprotein $\mathrm{E}$ by gene amplification and cleavage with HhaI. J. Lipid Res. 31:545548.

43. Gjone, E., and B. Bergaust. 1969. Corneal opacity in familial plasma cholesterol ester deficiency. Acta Ophthalmol. 47:222-227.

44. Schultz, J. R., E. L. Gong, M. R. McCall, A. V. Nichols, S. M. Clift, and E. M. Rubin. 1992. Expression of human apolipoprotein A-II and its effect on high density lipoproteins in transgenic mice. J. Biol. Chem. 267:21630-21636.

45. Subbaiah, P. V., R. A. Norum, and J. D. Bagdade. 1991. Effect of apolipoprotein activators on the specificity of lecithin:cholesterol acyltransferase: determination of cholesteryl esters formed in A-I/C-III deficiency. J. Lipid Res. 32:1601-1609.

46. Goldstein, J. L., and M. S. Brown. 1989. Familial hypercholesterolemia. In The Metabolic Basis of Inherited Diseases. 6th edition. C. R. Scriver, A. L. Beaudet, W. S. Sly, and D. Valle. McGraw Hill, Inc., New York. 1215-1250.

47. Williamson, R., D. Lee, J. Hagaman, and N. Maeda. 1992. Marked reduction of high density lipoprotein cholesterol in mice genetically modified to lack apolipoprotein A-I. Genetics. 89:7134-7138.

48. Nichols, W. C., R. E. Gregg, H. B. Brewer, Jr., and M. D. Benson. 1990. A mutation in apolipoprotein A-I in the Iowa type of familial amyloidotic polyneuropathy. Genomics. 8:318-323.

49. Gotoda, T., N. Yamada, T. Murase, M. Sakuma, M. Murayama, H. Shimano, K. Kozaki, J. J. Albers, Y. Yazaki, and Y. Akanuma. 1991. Differential phenotypic expression by three mutant alleles in familial lecithin:cholesterol acyltransferase deficiency. Lancet. 338:778-781.

50. Maeda, E., Y. Naka, T. Matozaki, M. Sakuma, Y. Akanuma, G. Yoshino, and M. Kasuga. 1991. Lecithin-cholesterol acyltransferase (LCAT) deficiency with a missense mutation in exon 6 of the LCAT gene. Biochem. Biophys. Res. Commun. 178:460-466.

51. Klein, H. G., P. Lohse, P. H. Pritchard, D. Bojanovski, H. Schmidt, and H. B. Brewer, Jr. 1992. Two different allelic mutations in the lecithin-cholesterol acyltransferase gene associated with the fish eye syndrome. J. Clin. Invest. 89:499-506.

52. Funke, H., A. von Eckardstein, P. H. Pritchard, J. J. Albers, J. J. P. Kastelein, C. Droste, and G. Assmann. 1991. A molecular defect causing fish eye disease: an amino acid exchange in lecithin cholesterol acyltransferase (LCAT) leads to the selective loss of $\alpha$-LCAT activity. Proc. Natl. Acad. Sci. USA. $88: 4855-4859$. 